\title{
Application of independent component analysis to Fermilab Booster
}

\author{
Xiaobiao Huang, ${ }^{1,2, *}$ S. Y. Lee, ${ }^{1}$ Eric Prebys, ${ }^{2}$ and Ray Tomlin ${ }^{2}$ \\ ${ }^{1}$ Department of Physics, Indiana University, Bloomington, Indiana 47405, USA \\ ${ }^{2}$ Fermi National Accelerator Laboratory, Box 500, Batavia, Illinois 60510, USA
}

(Received 22 March 2005; published 9 June 2005)

\begin{abstract}
Autocorrelation is applied to analyze sets of finite-sampling data such as the turn-by-turn beam position monitor (BPM) data in an accelerator. This method of data analysis, called the independent component analysis (ICA), is shown to be a powerful beam diagnosis tool for being able to decompose sampled signals into its underlying source signals. We find that the ICA has an advantage over the principle component analysis (PCA) used in the model-independent analysis (MIA) in isolating independent modes. The tolerance of the ICA method to noise in the BPM system is systematically studied. The ICA is applied to analyze the complicated beam motion in a rapid-cycling booster synchrotron at the Fermilab. Difficulties and limitations of the ICA method are also discussed.
\end{abstract}

DOI: 10.1103/PhysRevSTAB.8.064001

PACS numbers: 29.27.Bd, 41.75. $-\mathrm{i}, 29.85 .+\mathrm{c}$

\section{INTRODUCTION}

Data mining and analysis play an important role in uncovering underlying physics principles in complex dynamical systems. Using high precision closed orbit data of an accelerator storage ring with small knownperturbations, one can attain a vast data array of the orbit response matrix, which has been successfully applied to model and uncover problems associated with high intensity storage rings [1].

The model-independent analysis (MIA), an analysis of a massive data set obtained from beam position monitors (BPM) using a large number of pulses, can be used to untangle eigenmodes [2]. The spatial and temporal functions can be used to identify betatron motion and other sources of perturbations in linac transport systems. Recently, the method has been extended to study beam dynamics in high brightness storage rings using coherent rf dipole field excitation $[3,4]$.

The data sampled by BPMs reflect the beam transverse motion, which is a combination of betatron motion, synchrotron motion (coupled through dispersion) and perturbations from other sources, such as noise, ground motion, vibration source, wake field, etc. If the BPM system is linear, the sampled data can be considered as a linear mixture of a few physical source signals. The ultimate goal of data analysis is to uncover these independent source signals.

The MIA is essentially a principal component analysis (PCA), which tries to find a linear transformation of the samples to uncover the maximum amount of variance in the least number of uncorrelated components. Only when these source modes are weakly coupled or nondegenerate (unequal eigenvalues), can the MIA properly isolate these independent modes. When these conditions are met, the

\footnotetext{
*Electronic address: xiahuang@fnal.gov xiahuang@indiana.edu
}

MIA can be successfully used to identify various beam modes. When eigenmodes are coupled, one needs to apply narrowband filtering to isolated relevant modes [4].

For high intensity rapid-cycling accelerators, the source modes are normally found to be strongly coupled. For example, the turn-by-turn beam position monitor (BPM) data obtained from the Fermilab Booster, which accelerates high intensity proton bunches at $15 \mathrm{~Hz}$ from $400 \mathrm{MeV}$ to $8 \mathrm{GeV}$ beam kinetic energies, show that the synchrotron motion is strongly coupled to the betatron modes. The contaminated signal data should be removed by averaging before the PCA analysis can be properly carried out. Even so, a global perturbing signal can severely degrade the betatron modes, and other source signals can still leak into the betatron modes.

The independent component analysis (ICA) methods provide a remedy for MIA's limitation by identifying the independent source signals from the samples using unequal time correlations. Once identified, The source signals can provide information on the betatron and synchrotron motion and other perturbation modes according to their spatial and temporal functions. This new method is more immune to mode mixing and noise signals. Because of its ability in mode isolation, the ICA can be used to identify and study the unknown beam motions due to various perturbing sources. Implementation of ICA algorithms depends on the nature of the source signals [5-7]. The time-correlation based method is particularly efficient in isolating narrowband source modes and is used in this study.

Turn-by-turn BPM data of a synchrotron are normally composed of coupled betatron and synchrotron oscillations, contaminated with noises. If the transfer function of the BPM system is linear, the BPM readings can be considered as a linear combination of contributions of all physical processes. These contributions, each driven by a certain physical cause, are referred to as source signals. It would be convenient to study the physical sources if we could separate these source signals from the raw data. Both 
the MIA [2] and the ICA [7] are model independent in the sense that no a priori knowledge of the underlying physical processes is assumed.

The ICA method assumes that source signals have nonoverlapping power spectra, which often holds because the signals are usually harmonic oscillation with different tunes. The assumption assures that the unequal timecorrelation matrices of the source signals are diagonal. The demixing matrix, which transforms the sample data vector to the source signal vector is found as the joint diagonalizer of the unequal time-correlation matrices of the sample data with selected time-lag constants. Since the source modes can be isolated, their origin can be identified. Some of these modes in accelerator physics are horizontal and vertical betatron and synchrotron oscillations. These modes enable us to study not only the betatron motions but also linear coupling and synchrotron motion from BPM data.

This paper is intended to study the applicability and limitations of the ICA method in accelerators. We organize this paper as follows. In Sec. II, we introduce the ICA method, and demonstrate the applicability of ICA by applying it to analyze simulated data derived from simple models, and a model of tracking data obtained by the MAD program of the Fermilab Booster. We also explore the limitation of all finite data sampling methods. In Sec. III, we apply ICA to experimental data of the FNAL Booster taken under the condition of the normal ramping cycle. Betatron tunes are measured and compared to model calculation throughout the cycle [8]. The synchrotron tune is also measured and compared to direct measurements [9]. Beta functions and phase advance are also measured. Finally we use the result to correct the Fermilab Booster MAD model [10]. The conclusion will be addressed in Sec. IV.

\section{THE ICA DATA ANALYSIS METHOD}

The linear response of a dynamical system is represented by the relation between the $m$-dimensional observation vector $\mathbf{X}(t)$ and the $n$-dimensional source signal vector $\mathbf{s}(t)$ by

$$
\mathbf{X}(t)=\mathbf{A s}(t)+\mathcal{N}(t)
$$

where $\mathbf{A} \in \Re^{m \times n}$ is the mixing matrix with $m \geq n$ ( $n$ is unknown a priori) and $\mathcal{N}(t)$ is the noise vector, assumed to be stationary, zero mean, temporally white, and statistically independent of the source signal $\mathbf{s}(t)$. The task is to determine both the mixing matrix $\mathbf{A}$ and the source signals $\mathbf{s}(t)$ from the sample signal $\mathbf{X}(t)$.

For most of the physical processes, the source signals are assumed to be mutually independent and temporally correlated, i.e., the time-lagged covariance matrix $\mathbf{C}_{\mathbf{s}}(\tau) \equiv$ $\left\langle\mathbf{s}(t) \mathbf{s}(t+\tau)^{T}\right\rangle \quad$ is diagonal, $\quad$ i.e., $\quad\left\langle\mathbf{s}_{\mathbf{i}}(t) \mathbf{s}_{\mathbf{j}}(t+\tau)^{T}\right\rangle=$ $S_{i}(\tau) \delta_{i j}$. Here $\langle\cdots\rangle$ stands for mathematical expectation and $\tau$ is the time-lag constant. The source signal separation of the ICA method jointly diagonalizes the covariance matrices with selected time-lag constants $\tau$ and uses data whitening as a necessary preprocessing procedure. The algorithm that we use is listed as follows [5,7].

First, compute the $m \times m$ sample covariance matrix $\mathbf{C}_{\mathbf{X}}(0) \equiv\left\langle\mathbf{X}(t) \mathbf{X}(t)^{T}\right\rangle$. Perform singular value decomposition (SVD) on $\mathbf{C}_{\mathbf{X}}(0)$ to obtain

$$
\mathbf{C}_{\mathbf{X}}(0)=\left(\mathbf{U}_{1}, \mathbf{U}_{2}\right)\left(\begin{array}{cc}
\boldsymbol{\Lambda}_{1} & 0 \\
0 & \boldsymbol{\Lambda}_{2}
\end{array}\right)\left(\begin{array}{l}
\mathbf{U}_{1}^{T} \\
\mathbf{U}_{2}^{T}
\end{array}\right)
$$

where $\boldsymbol{\Lambda}_{1}, \boldsymbol{\Lambda}_{2}$ are diagonal matrices with $\min \left(\operatorname{diag}\left[\boldsymbol{\Lambda}_{1}\right]\right) \geq$ $\lambda_{c}>\max \left(\operatorname{diag}\left[\boldsymbol{\Lambda}_{2}\right]\right) \geq 0, \lambda_{c}$ is a cutoff threshold set to remove the singularity of the data matrix, and $\Lambda_{1}$ is $n \times n$ diagonal matrix with eigenvalues $\lambda_{1} \geq \lambda_{2} \geq \cdots \geq \lambda_{n} \geq$ $\lambda_{c}$. Using the matrix

$$
\mathbf{V} \equiv \boldsymbol{\Lambda}_{1}^{-1 / 2} \mathbf{U}_{1}^{T}
$$

we construct an $n$-component vector as $\boldsymbol{\xi}=\mathbf{V X}$. The vector $\boldsymbol{\xi}$ is called white because $\left\langle\boldsymbol{\xi} \boldsymbol{\xi}^{T}\right\rangle=\mathbf{I}$, where $\mathbf{I}$ is the $n \times n$ identity matrix. This step reduces the dimension of the data space, reduces the noise in the original data, and decorrelates and normalizes the data to facilitate the next step.

For a selected set of time-lag constants $\left\{\tau_{k}\right\}$ ( $k=$ $1,2, \ldots, K)$, compute the time-lagged covariance matrices $\left\{\mathbf{C}_{\xi}\left(\tau_{k}\right)=\left\langle\boldsymbol{\xi}(t) \boldsymbol{\xi}\left(t+\tau_{k}\right)^{T}\right\rangle\right\}$, form symmetric matrices $\overline{\mathbf{C}}_{\boldsymbol{\xi}}\left(\tau_{k}\right)=\left(\mathbf{C}_{\xi}\left(\tau_{k}\right)+\mathbf{C}_{\xi}\left(\tau_{k}\right)^{T}\right) / 2$, and find a unitary matrix $\mathbf{W}$ that diagonalizes all matrices $\overline{\mathbf{C}}_{\boldsymbol{\xi}}\left(\tau_{k}\right)$ of this set, i.e., $\overline{\mathbf{C}}_{\boldsymbol{\xi}}\left(\tau_{k}\right)=\mathbf{W D}_{\mathbf{k}} \mathbf{W}^{T}$, where $\mathbf{D}_{\mathbf{k}}$ is diagonal. In practice, joint diagonalization can be achieved only approximately. Algorithms for approximate joint diagonalization can be found in Ref. [11].

Finally, the source signals and the mixing matrix are $\mathbf{s}=$ $\mathbf{W}^{T} \mathbf{V X}$ and $\mathbf{A}=\mathbf{V}^{-1} \mathbf{W}$, respectively, where $\mathbf{V} \equiv$ $\boldsymbol{\Lambda}_{1}^{-1 / 2} \mathbf{U}_{1}^{T}$ and $\mathbf{V}^{-1}=\mathbf{U}_{1} \boldsymbol{\Lambda}_{1}^{1 / 2}$.

For digitized sample data $X_{i}(t)$, the time-lag constants $\tau_{k}$ are discrete integers. The expectation functional $\langle\cdots\rangle$ is replaced with sample average in practice. Improvements on the above algorithm have been studied by using robust whitening in Refs. [12,13], or a combination of nonstationarity and time-correlation algorithms in Ref. [12]. In accelerator application, we find that our algorithm is sufficient to isolate all independent signals.

The application of ICA to beam diagnosis involves three phases: data acquisition and preprocessing, source signal separation, and beam motion identification. To gain more information of the beam lattice, the beam needs to undergo coherent transverse motion in the time when the turn-byturn data are taken. A pinger or rf resonant excitation kicker should be fired once or periodically to excite the beam.

The data sampled by BPMs around the ring are put into a data matrix 


$$
\mathbf{X}=\left(\begin{array}{cccc}
x_{1}(1) & x_{1}(2) & \ldots & x_{1}(N) \\
x_{2}(1) & x_{2}(2) & \ldots & x_{2}(N) \\
\vdots & \vdots & \ddots & \vdots \\
x_{m}(1) & x_{m}(2) & \ldots & x_{m}(N)
\end{array}\right)
$$

where $N$ is the total number of turns, $m$ is the number of BPMs. The element $X_{i}(j)$ is the reading of the $i$ th BPM on the $j$ th turn. BPM gains may be applied to correct the BPM calibration error if necessary and available.

ICA algorithm is then applied to extract the mixing matrix $\mathbf{A}$ and source signals $\mathbf{s}$ from the data matrix $\mathbf{X}$. Each source signal $\mathbf{s}_{i}$ and its spatial distribution $\mathbf{A}_{\mathbf{i}}$, where $\mathbf{A}_{\mathbf{i}}$ is the $i$ th column of $\mathbf{A}$, is called a mode. The physical origin of a mode can be identified by its spatial and temporal functions.

An oscillating signal (e.g., betatron oscillation) that has a different phase at each BPM will appear as two modes with identical frequency spectrum. Coherent betatron motion excited by the pinger should be dampened by decoherence. An important signature of betatron modes is their tune. Let $\mathbf{u}(t)$ be the betatron oscillation component of the transverse motion, then

$$
\mathbf{u}(t)=\mathbf{A}_{\mathbf{b} \mathbf{1}} s_{b 1}(t)+\mathbf{A}_{\mathbf{b} 2} s_{b 2}(t),
$$

where $s_{b 1}(t)$ and $s_{b 2}(t)$ are sinelike and cosinelike modes, respectively. The betatron function and phase advance can thus be derived

$$
\begin{gathered}
\beta_{i}=a^{2}\left(A_{b 1, i}^{2}+A_{b 2, i}^{2}\right), \\
\psi_{i}=\tan ^{-1}\left(\frac{A_{b 1, i}}{A_{b 2, i}}\right),
\end{gathered}
$$

where $a$ is a constant depending on initial conditions. The fractional part of the betatron tune can be obtained by the fast Fourier transform (FFT) of the temporal function.

The synchrotron mode can be recognized because its temporal pattern reflects the synchrotron oscillation of momentum deviation $\Delta p / p_{0}=\delta(t)$. The spatial pattern of the synchrotron mode is dispersion function. Let $\mathbf{v}(t)$ be the synchrotron oscillation component in the transverse motion:

$$
\mathbf{v}(t)=\mathbf{A}_{s} s_{s}(t) .
$$

Note that there is only one mode because the synchrotron tune is much smaller than 1 . The dispersion $D$ and the synchrotron coordinate $\delta(t)$ are related by

$$
\begin{gathered}
\mathbf{D}=b \mathbf{A}_{s}, \\
\delta(t)=s_{s}(t) / b,
\end{gathered}
$$

with a constant $b$. The constant $a$ can be "determined" by the calibration of kicker strength and the "modeled" $\beta$ function at the kicker location through several kicker strengths. The constant $b$ can be calibrated through simultaneous phase measurement for the synchrotron motion.

In the presence of linear coupling, betatron modes have two separate eigenfrequencies $\left(\nu_{+}\right.$and $\left.\nu_{-}\right)$. Here, Eqs. (6) and (7) are still applicable. Higher order resonances, if they appear, can be recognized by their characteristic frequencies. Other signals can also occur. For example, ripples of magnet power supply can modulate the beam transverse motion. BPM cables and electronics may pick up an $\mathrm{rf}$ signal of nearby equipment. Some BPMs may insert an artificial signal to their output due to circuit malfunction. These signals can be identified and studied. The unidentified remainder of the original sample signals may be considered as random noise of the BPM system.

If transverse motion for both the horizontal (x) and vertical (z) planes can be recorded simultaneously, one can put them together in a matrix $\mathbf{y}=\left(\begin{array}{l}\mathbf{x} \\ \mathbf{z}\end{array}\right)$ for analysis. Linear coupling, including coupling angle between the horizontal and vertical planes, can be measured. The spatial function can be used to identify the source of linear coupling.

\section{A. The simulation model with linear coupling}

We study an ideal lattice model with known analytic solution. Let the equations of motion be

$$
\begin{aligned}
& \frac{d^{2}}{d \theta^{2}} x+\nu_{x}^{2} x+C z=0, \\
& \frac{d^{2}}{d \theta^{2}} z+\nu_{z}^{2} z+C x=0,
\end{aligned}
$$

where $\nu_{x}, \nu_{z}$ are betatron tunes, $C$ is the coupling constant, and $\theta=2 \pi f_{0} t$ is the orbital angle with revolution frequency $f_{0}$. The model assumes continuous, uniform focusing with linear coupling. The solution of the coupled equations is

$$
\begin{aligned}
& x=A_{1} \cos \nu_{+} \theta+A_{2} \cos \nu_{-} \theta, \\
& z=B_{1} \cos \nu_{+} \theta+B_{2} \cos \nu_{-} \theta,
\end{aligned}
$$

where the eigentunes are given by

$$
\nu_{ \pm}^{2}=\frac{1}{2}\left(\nu_{x}^{2}+\nu_{z}^{2} \pm \sqrt{\left(\nu_{x}^{2}-\nu_{z}^{2}\right)^{2}+4 C^{2}}\right) .
$$

The coefficients are determined by initial conditions. For $x(0)=x_{0}, x^{\prime}(0)=0, z(0)=z_{0}$, and $z^{\prime}(0)=0$, they are 
given by

$$
\begin{aligned}
& A_{1}=\frac{C z_{0}+\left(\nu_{x}^{2}-\nu_{-}^{2}\right) x_{0}}{\nu_{+}^{2}-\nu_{-}^{2}}, \\
& A_{2}=-\frac{C z_{0}+\left(\nu_{x}^{2}-\nu_{+}^{2}\right) x_{0}}{\nu_{+}^{2}-\nu_{-}^{2}}, \\
& B_{1}=\frac{C x_{0}+\left(\nu_{z}^{2}-\nu_{-}^{2}\right) z_{0}}{\nu_{+}^{2}-\nu_{-}^{2}}, \\
& B_{2}=-\frac{C x_{0}+\left(\nu_{z}^{2}-\nu_{+}^{2}\right) z_{0}}{\nu_{+}^{2}-\nu_{-}^{2}} .
\end{aligned}
$$

We generate multiple BPM turn-by-turn data so that we can compare the results of PCA and ICA with the analytic solution. We place $M$ BPMs uniformly around the ring, i.e., the phase advances at the $i$ th $(i=0,1, \cdots, M-1)$ BPM are $\psi_{+, i}=2 \pi \nu_{+} i / M$ and $\psi_{-, i}=2 \pi \nu_{-} i / M$. The readings this BPM will record are

$$
\begin{aligned}
& x_{i}(n)=A_{1} \cos \left(\nu_{+} n+\psi_{+, i}\right)+A_{2} \cos \left(\nu_{-} n+\psi_{-, i}\right), \\
& z_{i}(n)=B_{1} \cos \left(\nu_{+} n+\psi_{+, i}\right)+B_{2} \cos \left(\nu_{-} n+\psi_{-, i}\right),
\end{aligned}
$$

where $n$ is the revolution index. We can introduce badBPM modes and add white Gaussian random noises to each individual BPM to simulate the effect of noises.

\section{The demixing}

With the linear coupling model, each BPM detects a mixture of the normal modes ("+" and "-" modes, referring to signals with frequency $\nu_{+}$and $\nu_{-}$, respectively). It is desirable to get the pure + modes and modes. Since the betatron phase at each BPM is different, there are two + and two - modes. Using the spatial function of these modes, we can calculate the coefficients $A_{1}, A_{2}, B_{1}, B_{2}$ and the phase advances of the + mode and - mode. The coefficients allow us to derive beta functions and the coupling angle. Putting both horizontal and vertical data in one matrix is a necessary step in achieving mode demixing.

In our simulation studies we apply both PCA and ICA methods to compare their ability in mode separation. We find that the PCA method can separate the modes in most cases when the singular values (SV) of the + and the modes are substantially different from each other. However, when the SVs approach each other, PCA always produces modes with mixing. The closer the SVs are, the stronger the mixing is. On the other hand, ICA does not show any dependence on the relative magnitudes of the SVs. Figure 1 shows the spatial functions and tunes of modes 1 and 3 derived from the ICA and PCA, respectively. We note that the SVs of these modes are about equal, and the resulting PCA modes are mixed.
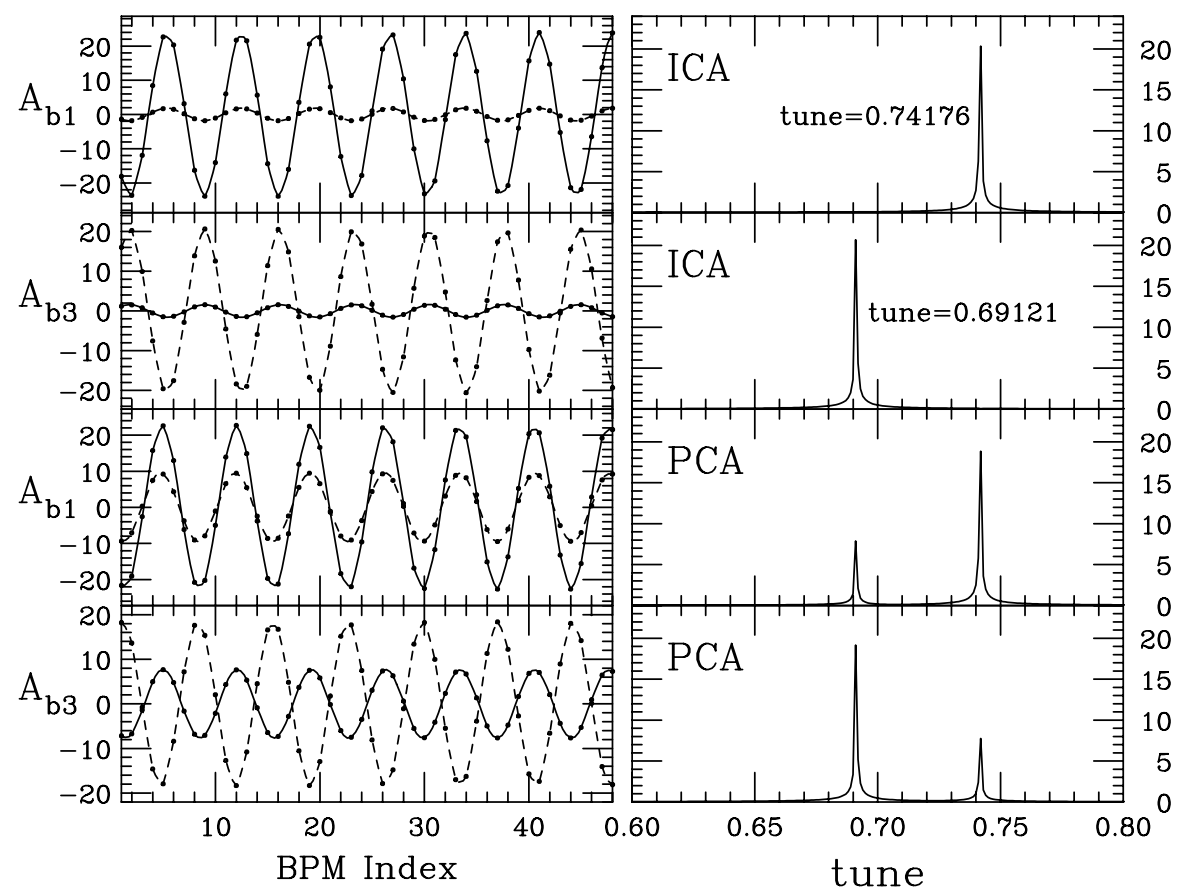

FIG. 1. The top and the second row are ICA modes of the linear coupling (LC) model. Model parameters are $\nu_{x}=6.74149, \nu_{z}=6.69149, C=0.05, x_{0}=y_{0}=1.0 A_{1}=1.0682, A_{2}=$ $-0.0682, B_{1}=0.0791, B_{2}=0.9209$. Top row: the spatial function and FFT of one of the + mode. The second row: the spatial pattern and FFT of one of the - mode. The horizontal (solid) and vertical (dash) curves in left plots are spatial functions. The right plots show FFT spectra of the temporal functions. The third and the bottom rows: modes 1 and 3 of the LC model using the PCA analysis. The SVs of the modes 1 and 3 are 122.0 and 98.8, respectively. 
What PCA essentially does is to decorrelate the sample data, i.e., to find an orthogonal basis to reinterpret the sample data. Although there are numerous transformations that decorrelate the sample data, the result of PCA is unique because PCA make sure the first component has the largest variance (corresponding to the first SV) and the second component has second largest variance and so on. The unique result of PCA is determined by the variances of the components, or the relative strength of the signals, which obviously does not concern the signals' other properties such as their power spectra or probability density functions. For example, let $s_{1}$ and $s_{2}$ be two normalized independent signals with $\left\langle s_{1} s_{1}\right\rangle=\left\langle s_{2} s_{2}\right\rangle=1,\left\langle s_{1} s_{2}\right\rangle=$ $\left\langle s_{1} s_{2}\right\rangle=0$. Let the data matrix be given by $x_{1}=2 s_{1}+$ $s_{2}$ and $x_{2}=s_{1}+2 s_{2}$. We can use a set of new basis vectors $w_{1}=(1 / \sqrt{2})\left(s_{1}+s_{2}\right)$ and $w_{2}=(1 / \sqrt{2})\left(s_{2}-s_{1}\right)$ or any orthogonal transform as the basis vectors for the source signals. However, we prefer the basis $s_{1}$ and $s_{2}$ because they are independent. The additional condition that we impose is the independence of signals $s_{1}$ and $s_{2}$, i.e., $\left\langle s_{1}(t) s_{1}(t+\tau)\right\rangle=S_{1}(\tau), \quad\left\langle s_{2}(t) s_{2}(t+\tau)\right\rangle=S_{2}(\tau), \quad$ and $\left\langle s_{1}(t) s_{2}(t+\tau)\right\rangle=\left\langle s_{2}(t) s_{1}(t+\tau)\right\rangle=0$. The new basis will inevitably be found to be $s_{1}$ and $s_{2}$ when the autocorrelation condition is imposed.

In the linear coupling model, the sample of each BPM is a mixture of 4 source signals $s_{i}(t)(i=1,2,3,4)$ among which are two + modes that make a + mode subspace and the other two make a - mode subspace. The resulting basis s contains only 4 nontrivial components. The source signals are considered separated if two components of $\mathbf{s}$ are in the + subspace and the other two are in the - subspace. However if there is a component which crosses the + and - subspace, the modes are still mixed.

From the above discussion, we see that PCA does not guarantee separation of the source signals. The basis $\mathbf{w}$ vectors are orthogonal transformation of the source signal basis vectors $\mathbf{s}$ depending on the strengths of the source signals in the sample data. The result depends on the distribution of the components of $\mathbf{x}$ or $\mathbf{z}$ in the space spanned by $\mathbf{s}$. On the other hand, ICA makes use of the fact that the power spectra of source signals are distinct and the autocorrelation covariance matrices are diagonal to find the source signals.

\section{The effects of bad-BPM signals}

To further illustrate the behavior of PCA and ICA, we introduce a narrowband bad-BPM harmonic oscillation at a frequency far away from the betatron frequencies. This signal is added only to one BPM, i.e., the spatial vector of this mode is localized at a "bad" BPM. By changing the amplitude of this noise signal we can change the SV of this mode. We observed that as the SV of this bad-BPM mode is near that of the - modes or + modes, the mode mixing occurred. However the ICA mode is immune of such mode mixing. The top two rows of Fig. 2 show that the betatron and the narrowband noise modes are mixed in PCA, where
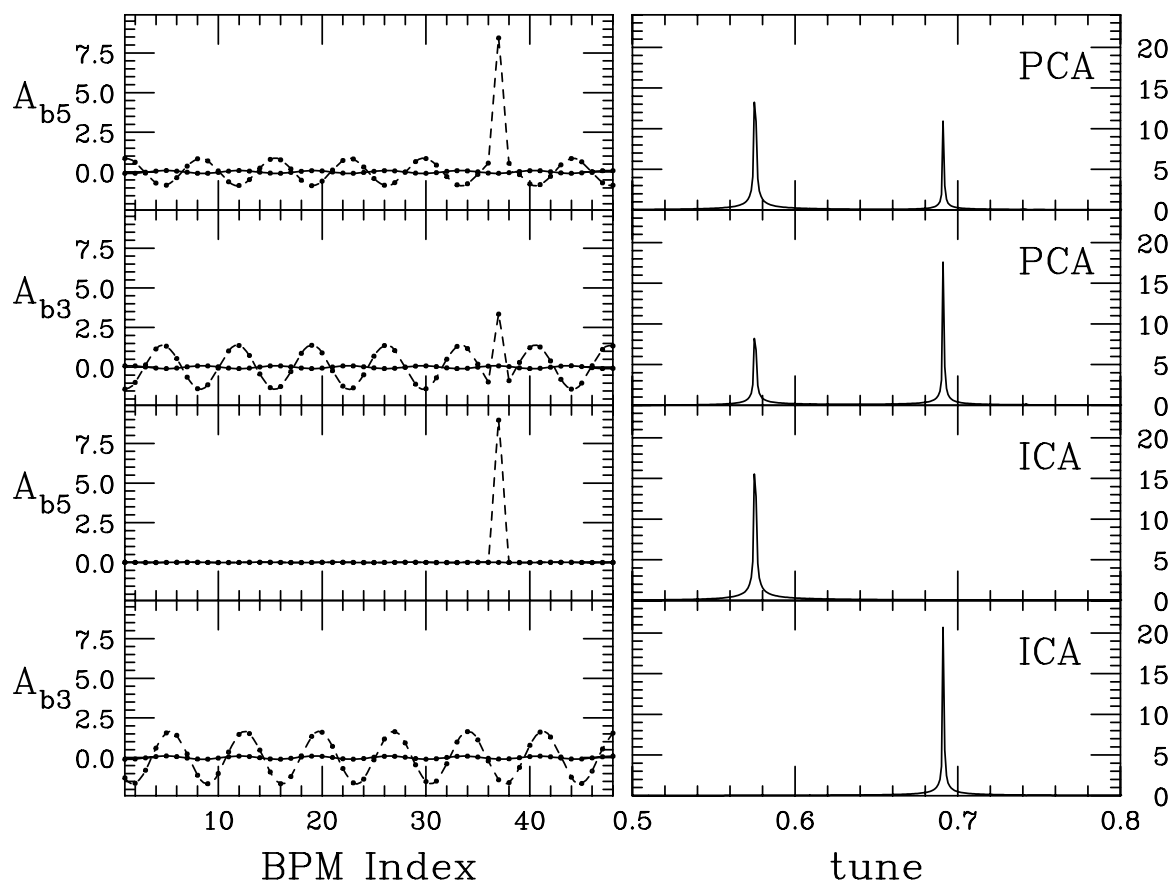

FIG. 2. PCA modes of the LC model with a localized bad-BPM signal. Model parameters: $\nu_{x}=6.74149, \nu_{z}=6.69149, C=0.05$, $x_{0}=1.0, y_{0}=0.0, A_{1}=0.9945, A_{2}=0.0055, B_{1}=0.0736, B_{2}=-0.0736$. A harmonic oscillation signal is added to BPM V37 with tune $\nu=0.57545$ and amplitude $D=0.4$. The top row: mode 3 with $\mathrm{SV}=9.4$. The second row: mode 5 with $\mathrm{SV}=7.5$. These two modes are mixed. the third and the fourth rows are ICA modes of the LC model with a bad-BPM harmonic oscillation signal. The localized "bad-BPM" mode is completely separated. 
the SVs are 9.4 and 7.5 for the betatron and noise modes, respectively. The bottom two rows of Fig. 2 show clearly that the ICA analysis is immune to mode mixing.

Another type of bad-BPM signal is white Gaussian noise. Applying the noise to a single bad BPM and adjusting the noise level so that its SV is about the same as that of the betatron mode, we find that the PCA modes are mixed again. The results for both PCA and ICA are shown in Fig. 3, where the mode mixing occurs for PCA if the SVs of these two modes are close to each other. In the PCA, the betatron mode has leaked into the bad-BPM mode as shown in the second row of Fig. 3. The ICA can easily isolate these two modes as shown in rows 3 and 4 of Fig. 3.

Since the PCA depends on the strengths of the source signals, it is sensitive to bad BPMs which are often noisy and strong. Thus it is absolutely necessary to exclude the bad BPMs before applying the PCA method. On the other hand, the ICA is more robust to bad BPMs. This is an advantage, especially for on line applications.

\section{The effects of low level noises}

In reality BPM readings always contain random noises which affect the results of data analysis. We insert white Gaussian noises into the simulation data matrix. The rms errors of the resulting beta functions and phase advances of both the ICA and PCA methods are estimated as shown in Fig. 4. The result agrees well with the analytic error estimation of PCA in Eq. (17) of Ref. [3]. It turns out that PCA and ICA have equal performances with respect to random noises. This is reasonable because ICA takes PCA as its first step (whitening) and white noises play little role in diagonalization of the unequal-time covariance matrices.

\section{The effects of number of turns}

Both PCA and ICA are subject to deficiencies due to the finite sampling points. Both methods assume diagonal covariance matrix of the source signals, which is true only asymptotically, i.e., the number of sampling points goes to infinity. In reality, the results of both PCA and ICA are affected by the number of sampling points. The offdiagonal elements of the covariance matrix of two harmonic signals can be estimated by

$$
\begin{aligned}
\left\langle s_{1}(t) s_{3}(t)\right\rangle & =\frac{1}{N_{t}} \sum_{n=0}^{N_{t}-1} \sin \left(2 \pi \nu_{+} n\right) \sin \left(2 \pi \nu_{-} n\right) \\
& \approx \frac{1}{2 N_{t}} \frac{\sin \pi \Delta \nu N_{t}}{\sin \pi \Delta \nu} \cos \pi \Delta \nu\left(N_{t}-1\right)
\end{aligned}
$$

where $s_{1}(t)$ and $s_{3}(t)$ are the two signals with tune $\nu_{+}$and $\nu_{-}$, respectively, $\Delta \nu=\nu_{+}-\nu_{-}$, and $N_{t}$ is the number of sampling points. The effects of $N_{t}$ to PCA and ICA can be simulated. Figure 5 shows the dependence of $\mathbf{C}_{\mathbf{s}}(1,3)=$ $\left\langle s_{1}(t) s_{3}(t)\right\rangle$ on the sampling turns $N_{t}$ and the resulting error
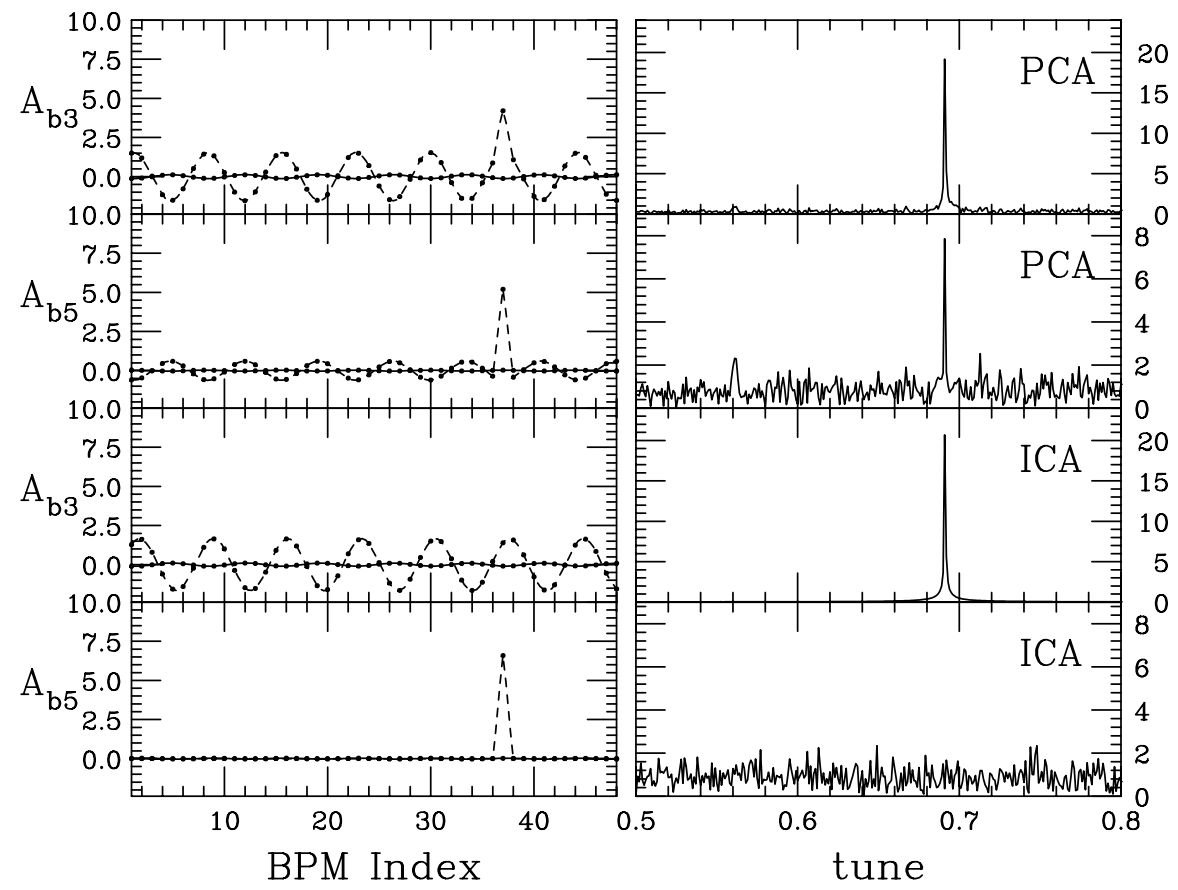

FIG. 3. The top and the second row are PCA modes of the LC model with a localized bad BPM with Gaussian white noise signal. Model parameters: $\nu_{x}=6.74149, \nu_{z}=6.69149, C=0.05, x_{0}=1.0, y_{0}=0.0 A_{1}=0.9945, A_{2}=0.0055, B_{1}=0.0736, B_{2}=$ -0.0736 . The signal added to BPM V37 is white Gaussian noise. The top row corresponds to mode 3 with SV $=8.4$, and the second row is the mode 5 with $\mathrm{SV}=6.1$. The two PCA modes are mixed. The third and the fourth rows are ICA modes of the LC model with a localized bad-BPM Gaussian white noise. The localized "bad-BPM" mode is completely separated. 


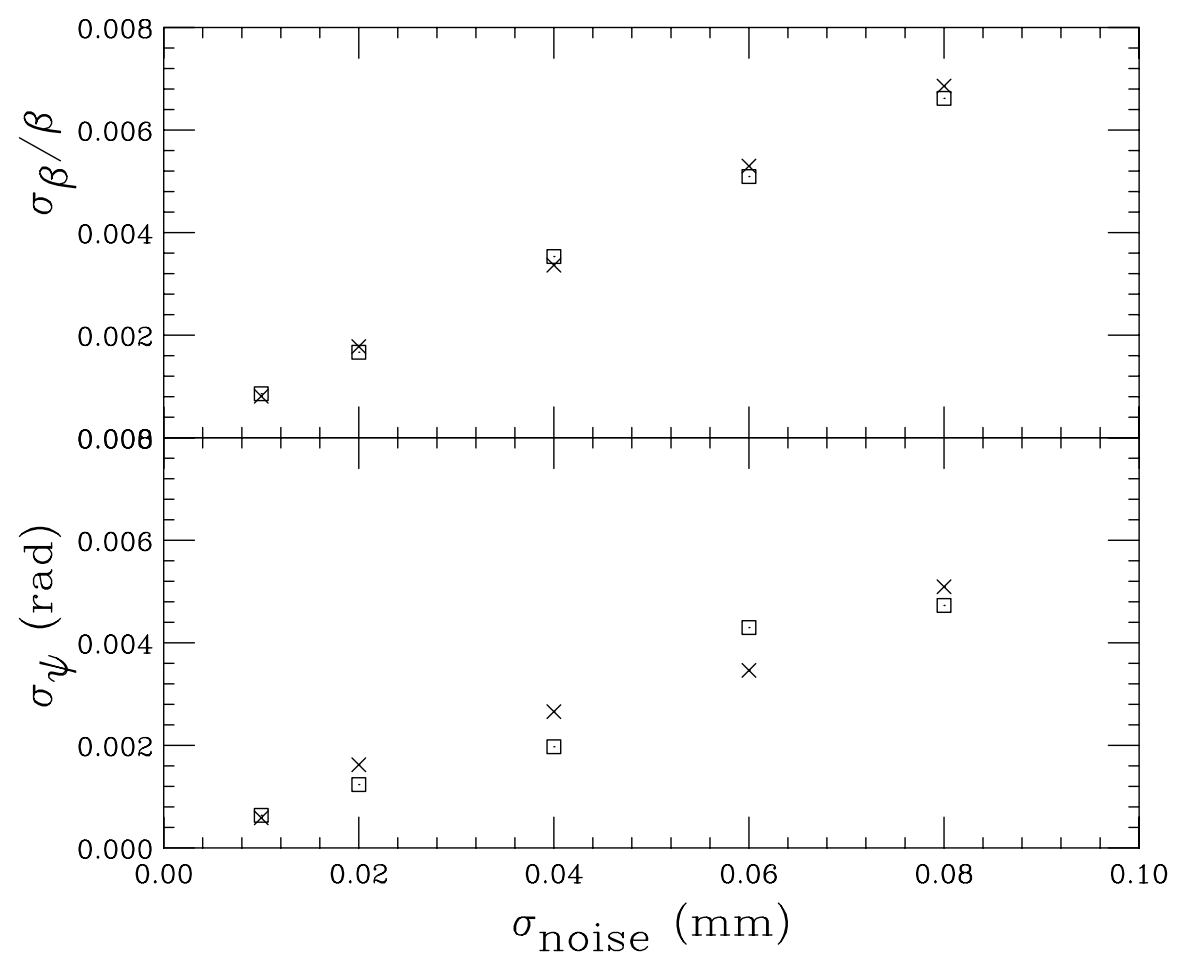

FIG. 4. Estimation of errors of ICA (cross) and PCA (square) methods with various random noise levels in the LC model. The model parameters are the same as Fig. 2. Data of 1000-turn are used to calculate $\sigma_{\beta} / \beta$ (left plot) and $\sigma_{\psi}$ (bottom plot). The estimation at each noise level $\sigma_{\text {noise }}(x$ axis) is made by repeating the measurement of $\beta$ and $\psi 10$ times with white Gaussian random noises added to each BPM.

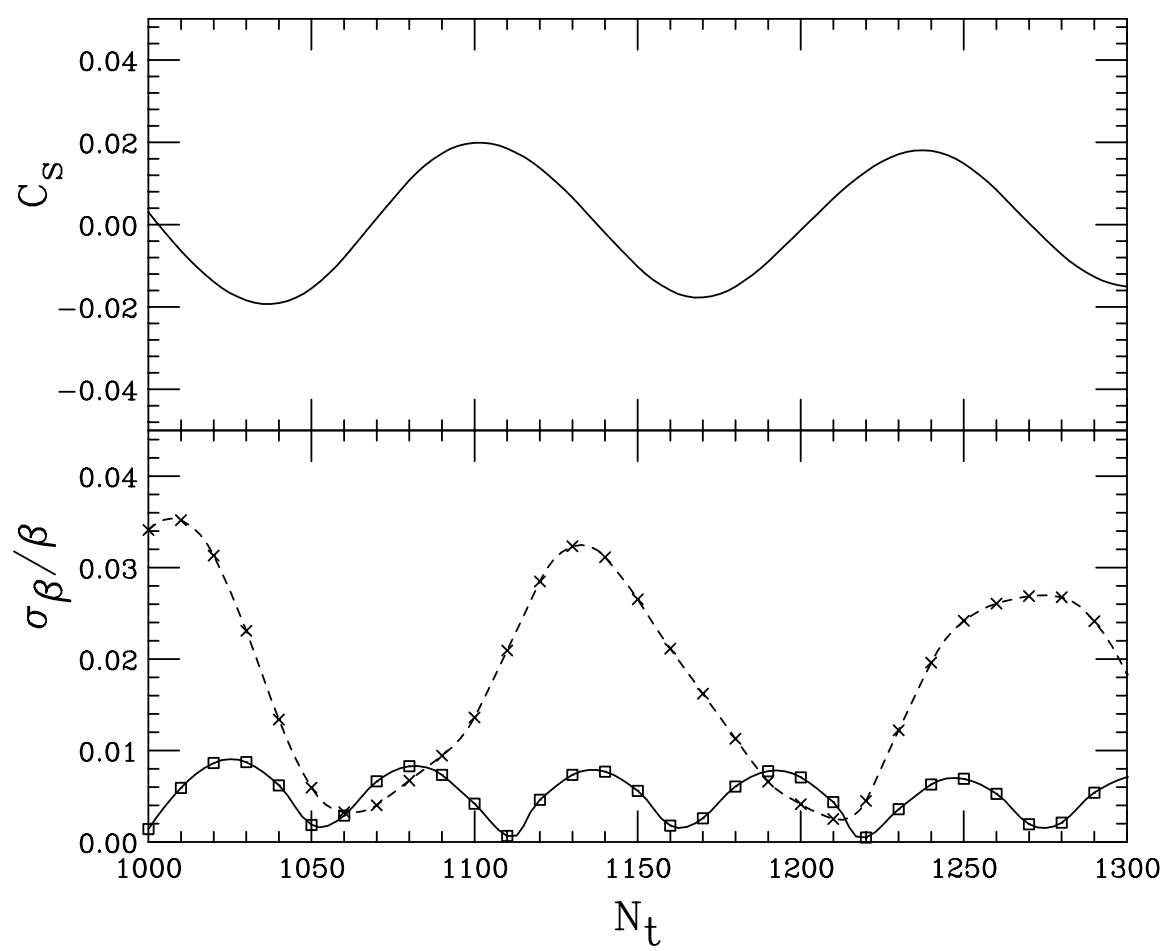

FIG. 5. Effect of number of the sampled turns $\left(N_{t}\right)$ in the LC model with $\nu_{+}=6.7447$ and $\nu_{-}=6.7372$. Top: off-diagonal elements of source signals $C_{s}(1,3)$. Bottom: $\sigma_{\beta} / \beta$ vs $N_{t}$ for ICA (solid) and PCA (dashed). 
in $\sigma_{\beta} / \beta$ with respect to $N_{t}$. The ICA method is less affected by $N_{t}$ because its results are based on diagonalization of several autocorrelated matrices instead of only one.

\section{B. Application to tracking data}

To explore the capability of ICA in actual data analysis, we employ the ICA technique to process BPM data produced by tracking programs such as MAD. In this model analysis, we have also compared and verified the salient features and limitations of the ICA and PCA methods as discussed in the previous section of a special solvable linear coupling model.

Since the ICA method can isolate beam modes, we employ this method to identify nonlinear resonance modes in tracking data. We find that higher order resonance signals can indeed be easily separated. Using the Fermilab Booster as an example, including excitation of sextupoles and skew quadrupoles, we can easily separate modes associated with resonances such as $\nu_{x} \pm \nu_{z}, 2 \nu_{x}$, $2 \nu_{z}, 2 \nu_{x} \pm \nu_{z}$, and $2 \nu_{x}-\nu_{z}$. Figure 6 shows an example of a third order resonance mode corresponding to $-\nu_{x}+$ $2 \nu_{z}-1=0$. Although the tracking data can be used to construct the Poincaré surface of section [14], the physical meaning of the spatial vector has not been fully understood. Since the signals of higher order resonance in the nominal operational condition of the Fermilab Booster are usually buried under the noise floor, we will not study its importance in this study. The ICA method may provide an alternative to the frequency map analysis as suggested in Ref. [15].

\section{ICA ANALYSIS FOR THE FERMILAB BOOSTER}

The main task of this paper is to use the ICA method in the study of beam dynamics at the Fermilab Booster. The Fermilab Booster is a fast ramping accelerator at $15 \mathrm{~Hz}$. The circumference is $474.2 \mathrm{~m}$. The betatron tunes are about $\nu_{x}=6.7$ and $\nu_{z}=6.8$. We applied the ICA analysis to the booster turn-by-turn BPM data taken in normal cycles, from which we derived betatron tunes, beta functions and phase advances at different stages of the cycle. We also separated out synchrotron signals which enable us to see the evolution of synchrotron tune in a normal cycle. At the normal operation settings, the booster was running with all of its correctors (trim dipoles, trim quads and skew quads, etc.) on. These settings, including their ramping components, were recorded to build a lattice model as realistic as possible.

Four data sets were taken with 1-turn injection and three were taken with 4-turn injection, corresponding to extraction intensity $0.5 \times 10^{12}$ and $2.0 \times 10^{12}$ protons per pulse, respectively. The horizontal pinger at the section L9 was fired periodically every $0.5 \mathrm{~ms}$ to excite beam transverse motion. The pinging strength was ramped up from $0.6 \mathrm{kV}$ at injection to $2.4 \mathrm{kV}$ at extraction to partially compensate the momentum increase.

The total $33.3 \mathrm{~ms}$ ramping cycle contains 20000 turns. Because the beam momentum changes swiftly, we divide the cycle into small pieces so that each piece (about 250 turns) contains one burst of transverse motion caused by the pinger.

\section{A. Betatron modes}

The betatron modes of a typical horizontal modes are shown in Fig. 7. Using the spatial function of the betatron modes in Eqs. (6) and (7), one obtains betatron amplitude functions and phase advances at each BPM. Figure 8 compared the measured betatron amplitude function with that obtained from MAD modeling.

The fractional part of the betatron tune can be derived from the FFT on the temporal function, shown in Fig. 9 for the entire ramping cycle. The betatron tunes calculated from the realistic model machine parameters are also shown as solid and dashed lines for comparison.

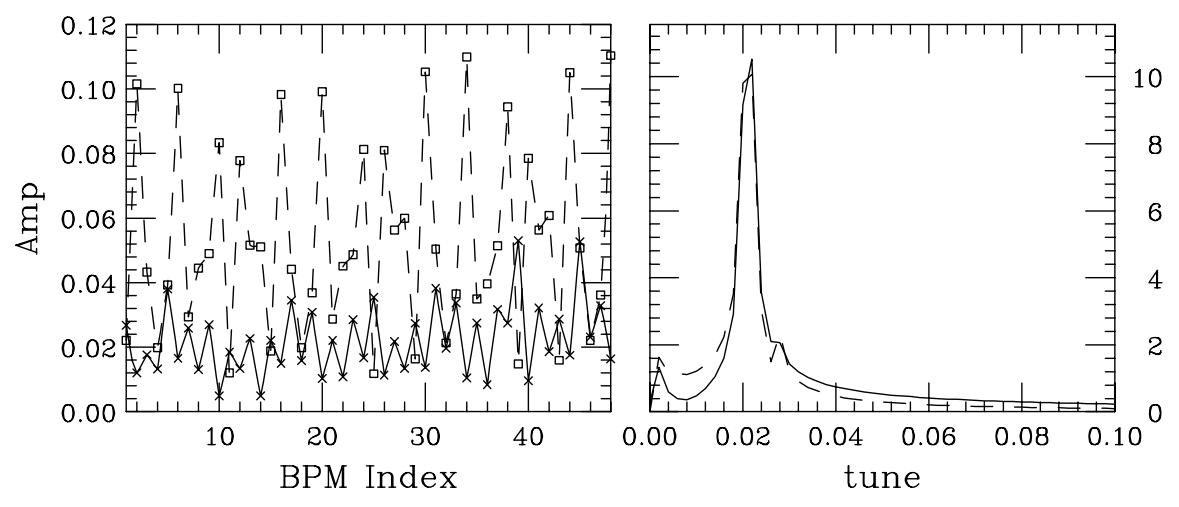

FIG. 6. Third order resonance signals corresponding to $-\nu_{x}+2 \nu_{z}-1=0$ in tracking data (500 turns) of the booster. The tune of the signals is 0.02107 , while $\nu_{x}=6.65753, \nu_{z}=6.83929$. The currents in sextupole families are ISEXTL $=20 \mathrm{~A}$, ISEXTS $=5 \mathrm{~A}$. Left plot: Amplitude of the resonance signal at horizontal (solid cross) and vertical (dash square) BPMs. Right plot: the FFT spectrum of the two resonance signals. 


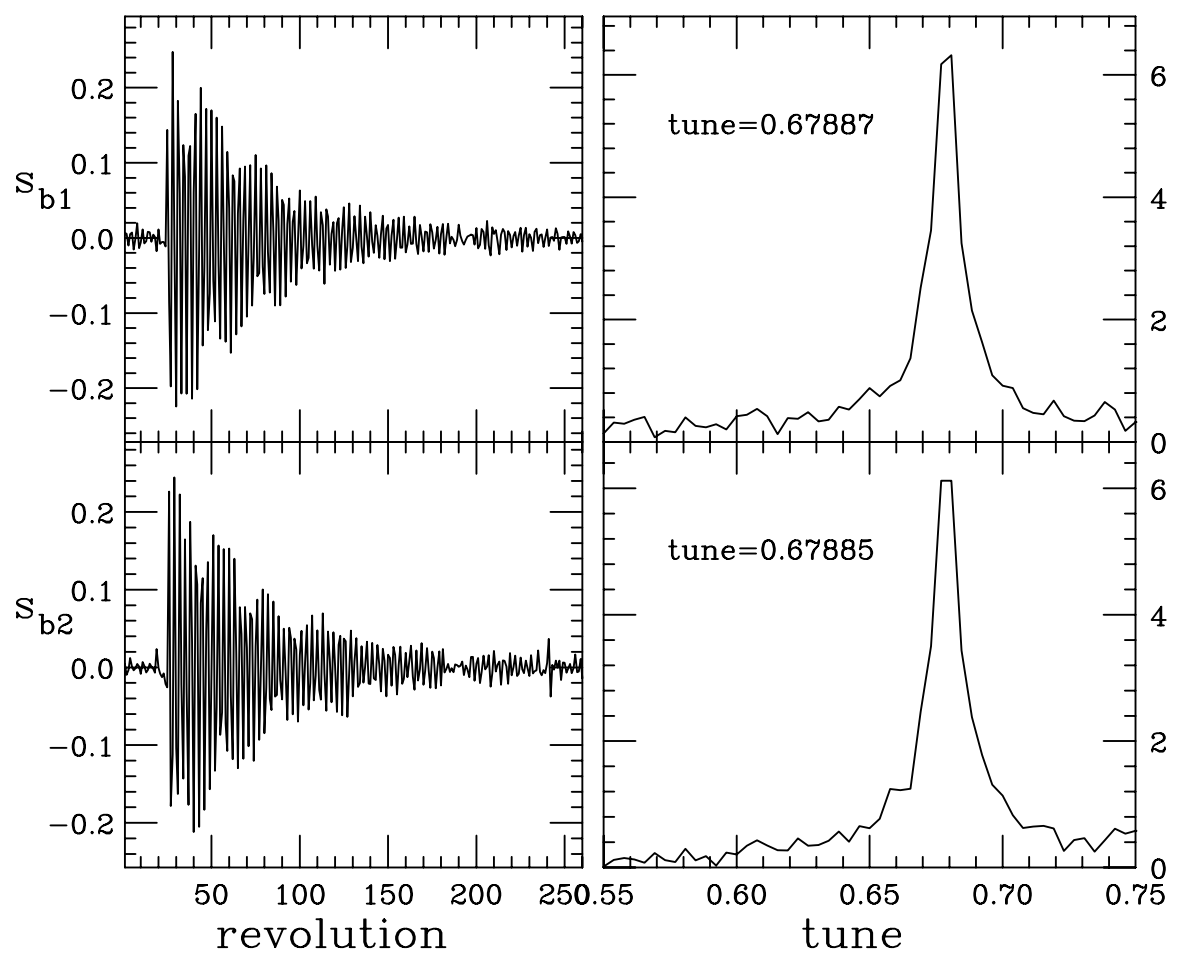

FIG. 7. Two modes of horizontal signal are shown on the left plots, and the corresponding FFT spectra are shown as the horizontal betatron tune. The ICA extracts a single betatron mode from data of all BPMs. The betatron mode is a purer sinusoidal signal and the tune evaluation method in Ref. [18] can be used to achieve higher precision. Note that the decoherence is not an independent signal, and thus cannot be isolated.

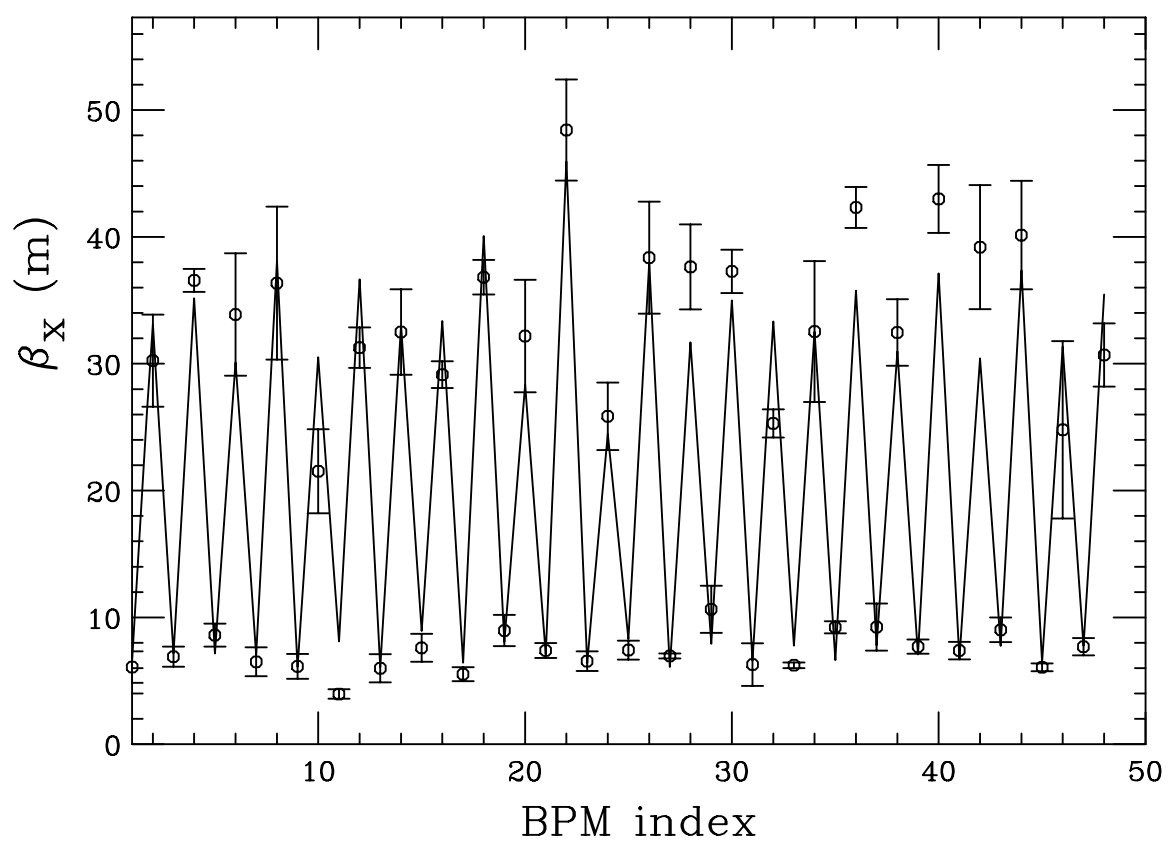

FIG. 8. Using the spatial function of two horizontal modes, one can calculate the betatron amplitude function at each BPM and phase advance between BPMs. The measured BPM is compared with the MAD model. The error bars were estimated with the standard deviation of the betatron function derived from the four data sets. 


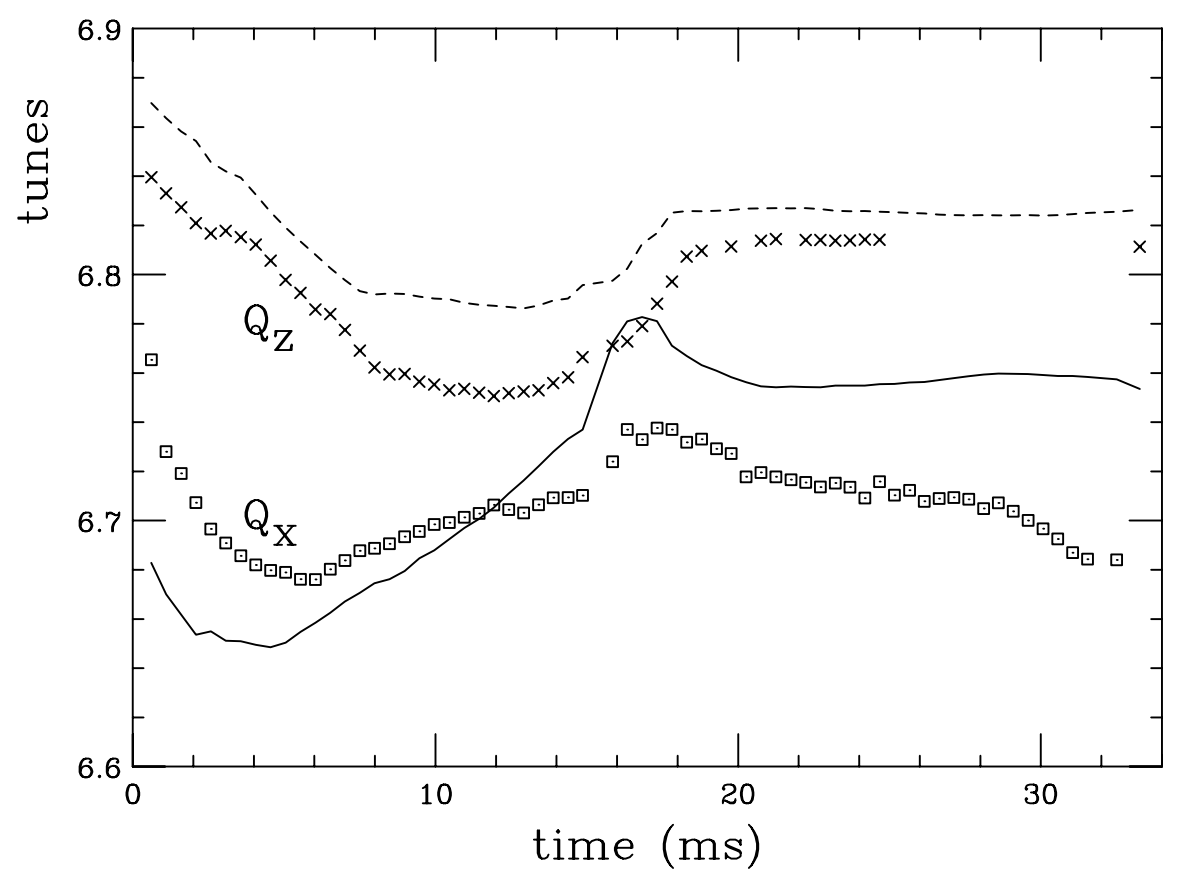

FIG. 9. The horizontal (square) and vertical (cross) betatron tunes in a booster cycle. Tunes calculated by MAD model are compared to measurements (solid and dashed lines).

\section{B. Synchrotron modes}

Dispersion function can be measured at the beginning and near the end of the cycle where large momentum deviation naturally occurs. The derived dispersion function is compared with the MAD modeling in Fig. 10. Similar agreement can be obtained in the orbit response matrix analysis [16]. The synchrotron tune is obtained from the
FFT of the temporal functions. Figure 11 shows the evolution of synchrotron tune in a cycle, excluding the transition crossing zone, where the synchrotron tune is nearly zero.

In the middle of the ramping cycle, the observed synchrotron modes are considerably weaker. There are two synchrotron modes, where their temporal patterns give the

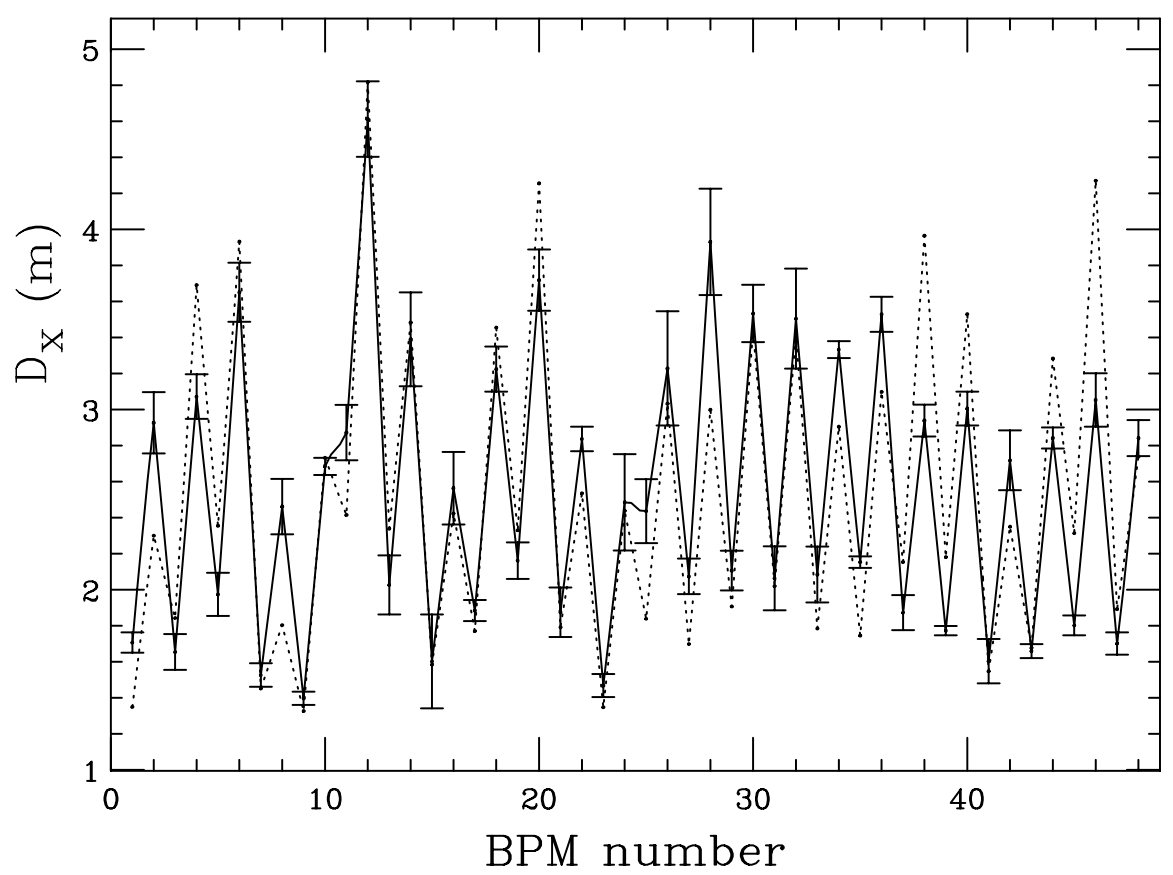

FIG. 10. The measured dispersion function is compared with the MAD modeling (dotted line) based on a realistic Fermilab Booster lattice. The error bars were estimated with the standard deviation of the dispersion function derived from the four data sets. 


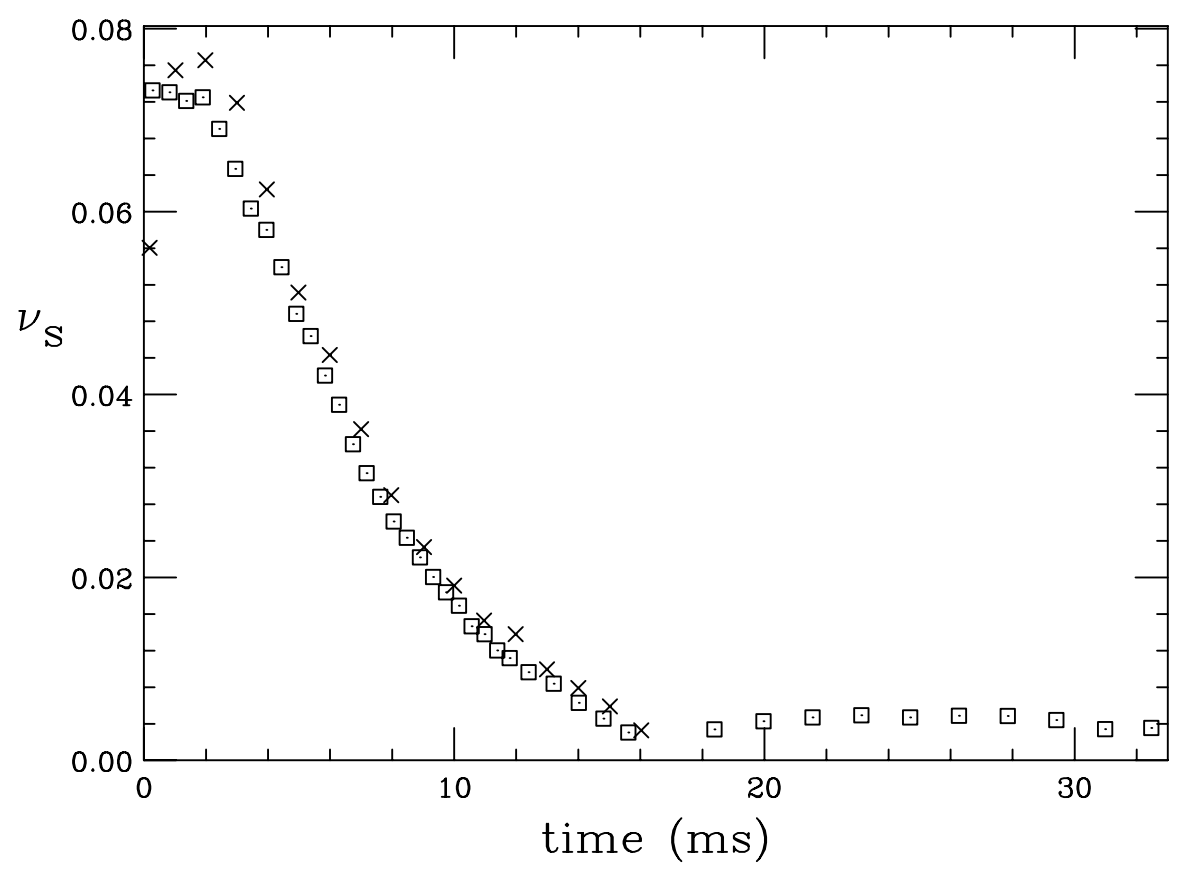

FIG. 11. The synchrotron tune in a booster cycle. The squares are measured from turn-by-turn data with ICA method. The crosses are measured from phase signal with synchrotron phase detector (SPD) as in Ref. [9]. Note that the SPD method has difficulty in measuring the synchrotron tune above the transition energy at around the $14.5 \mathrm{~ms}$.

synchrotron tune as shown in Fig. 11. However, their spatial functions often do not resemble the dispersion

$$
\Delta p / p(s, t)=\frac{1}{D(s)}\left(A_{s 1}(s) s_{1}(t)+A_{s 2}(s) s_{2}(t)\right)
$$
function. To understand these data, we show the mode function

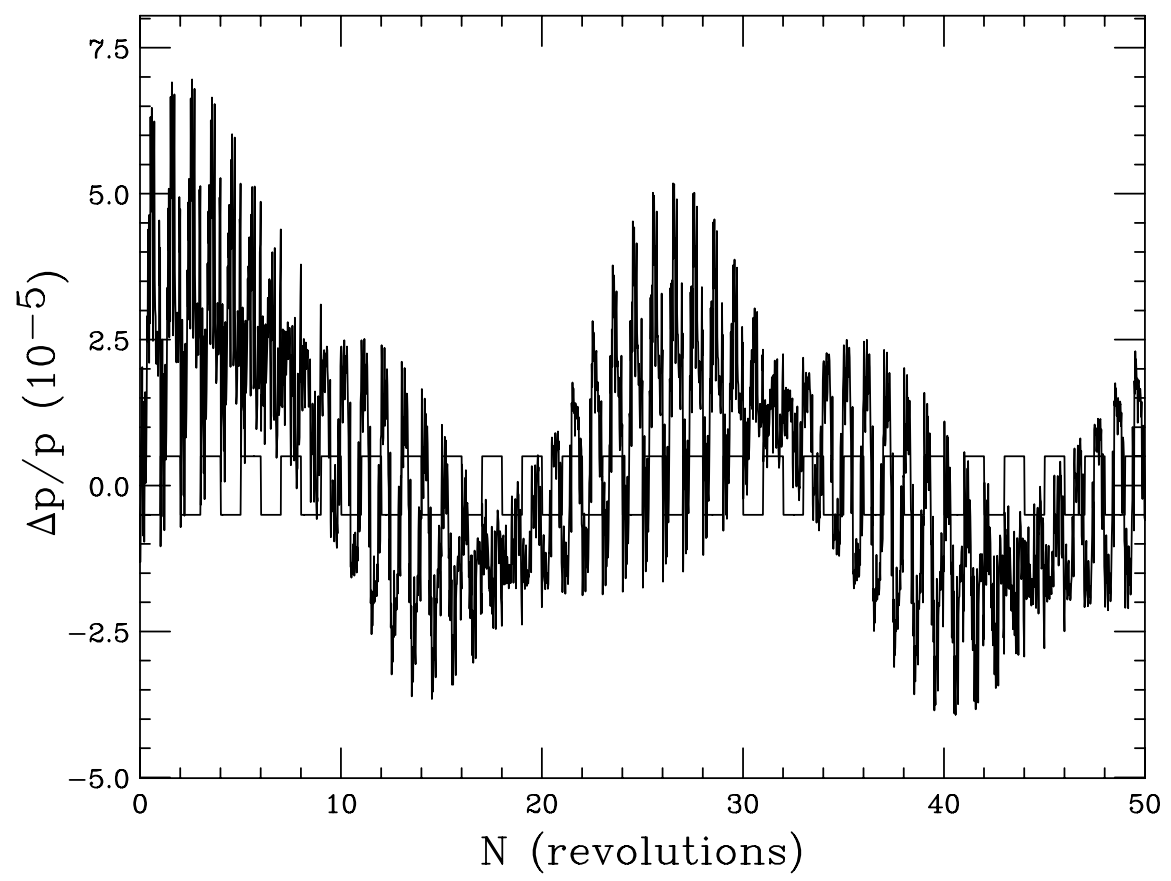

FIG. 12. The turn-by-turn data, divided by the dispersion function, of the synchrotron mode is plotted at each BPM for a total of 50 revolutions, starting from the revolution number 3001. The normalized vertical axis is equivalent to the fractional off-momentum variable. Each square box represents one revolution. 
where the each square box corresponds to one turn in the booster and $D(s)$ is the dispersion function shown in Fig. 10. Using the fact the $E \approx 1.855 \mathrm{GeV}$ at turn 3001 and amplitude of the energy oscillation is about $3.5 \times 10^{-5}$ as shown in Fig. 12, we estimate that the mode function has an amplitude of energy deviation of the order of $\Delta E=$ $\beta^{2} E\left(\Delta \hat{p} / p_{0}\right) \approx 50 \mathrm{keV}$. This agrees well with a recent estimate of the one-turn energy gain/loss of about $60 \mathrm{keV}$ in the longitudinal damper [17]. The one-turn energy gain at turn 3001 is about $340 \mathrm{keV}$, i.e., the average energy gain of each rf cavity is about 20 or $40 \mathrm{keV}$ per pair.

Figure 13 shows the spatial and temporal functions of two synchrotron modes. The behavior of the spatial pattern of synchrotron modes could be a consequence of (1) beam energy gain and loss by the longitudinal damper, (2) mismatched ramping curves of the energy gain in rf cavities and the dipole fields. Figure 14 shows the spatial function divided by the dispersion function. This can be thought of as the amplitude of the off-momentum coordinate at each BPM location. Since the beam energy changes rapidly by about $340 \mathrm{keV}$ in one revolution, each BPM can see different momentum deviation.

\section{MODELING THE BOOSTER}

The ICA method provides a means to measure the linear optics functions such as beta functions, phase advances, and dispersion. These functions can be used to correct the accelerator model (e.g., MAD) by tuning the model to minimize the difference between calculation and measure- ment. In the booster modeling, the available parameters are quadrupole gradients $K_{1}$ of the 96 main magnets.

We define the merit function

$$
\begin{aligned}
f(\mathbf{q}) & =\chi^{2}=\frac{1}{2} \sum_{i=1}^{240} r_{i}^{2}, \quad r_{i}=\frac{y_{i}(\mathbf{q})-y_{i}^{d}}{\sigma_{i}}, \\
\mathbf{y} & =\left(w_{1} \boldsymbol{\beta}_{x}, w_{2} \Delta \psi_{x}, w_{3} \boldsymbol{\beta}_{z}, w_{4} \Delta \psi_{z}, w_{5} \mathbf{D}_{x}\right),
\end{aligned}
$$

where $\mathbf{q}$ is a $96 \times 1$ vector consisting of the body quads corrections (i.e., $\Delta K_{1}$ ), $\boldsymbol{\beta}_{x}, \Delta \boldsymbol{\psi}_{x}, \boldsymbol{\beta}_{z}, \Delta \boldsymbol{\psi}_{z}, \mathbf{D}_{x}$ are all row vectors with 48 components at 48 BPM locations, $\mathbf{y}(\mathbf{q})$ and $\mathbf{y}^{d}$ are both $1 \times 240$ vectors containing the model and measured linear optics functions, respectively, $\sigma_{i}$ is the corresponding error estimation of $y_{i}^{d}$ serving as weight in the definition. The additional weights $w_{i}, i=1,2, \ldots, 5$ are used to account for our confidence over the five categories of fitting data. We may set them to $w_{1}=w_{3}=$ $w_{5}=1$ and $w_{2}=w_{4}=4$ to put more weights on the phase advances because they are independent of BPM calibration.

This nonlinear least square problem can be solved iteratively by Levenberg-Marquardt method. In each iteration we compute the Jacobian matrix defined as

$$
J_{i j}=\frac{\Delta y_{j}}{\Delta q_{i}}
$$

and solve

$$
\left(\mathbf{J}^{T} \mathbf{J}+\lambda \mathbf{I}\right) \Delta \mathbf{q}=-\mathbf{J}^{T} \mathbf{r}_{0}
$$

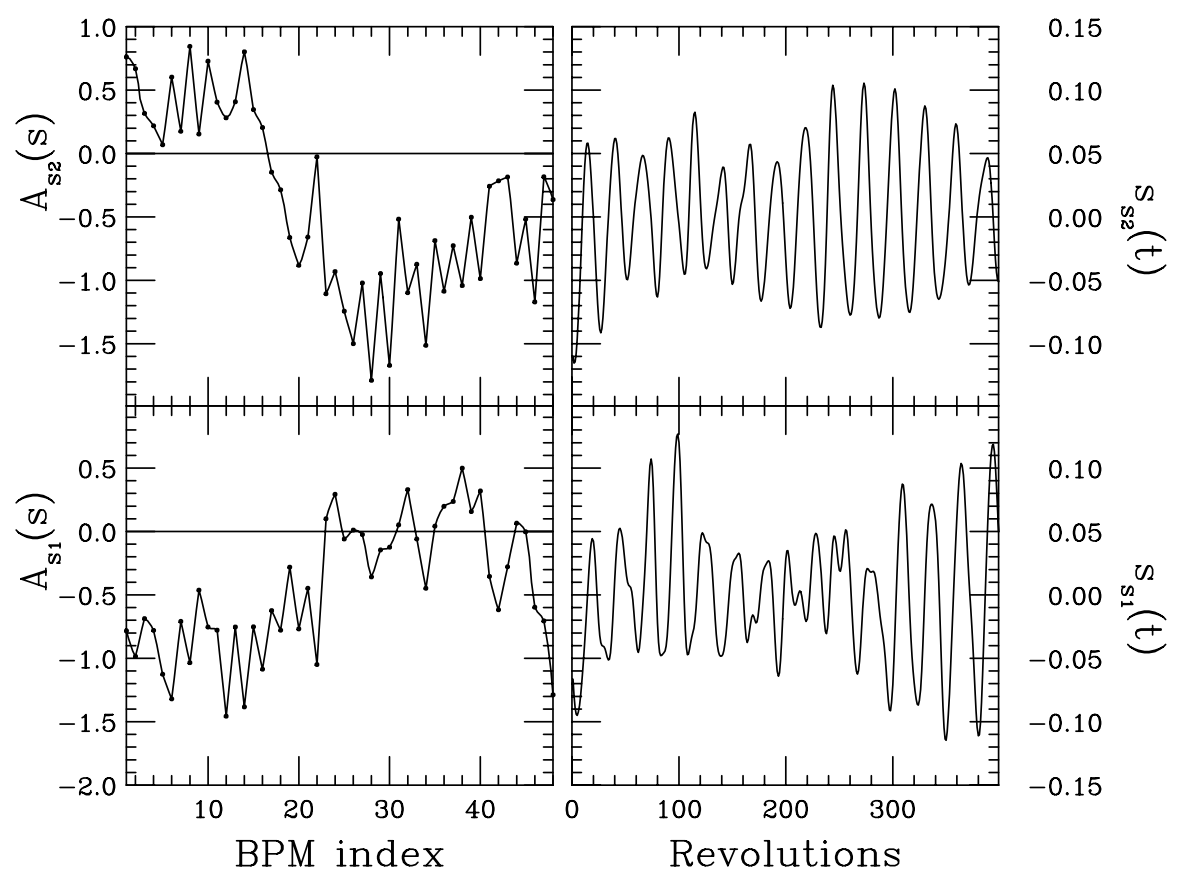

FIG. 13. Synchrotron modes in the middle of a booster cycle. The turn range is 3001 to 3400 (see Fig. 12). The temporal signals provide us the synchrotron tune shown in Fig. 11. The spatial pattern does not resemble the dispersion function. Furthermore, the spatial function crosses zero. The effective amplitude of the off-momentum coordinate is about $10^{-5}$. This may result from the longitudinal damper or mismatch between the beam energy and the main dipole field. 


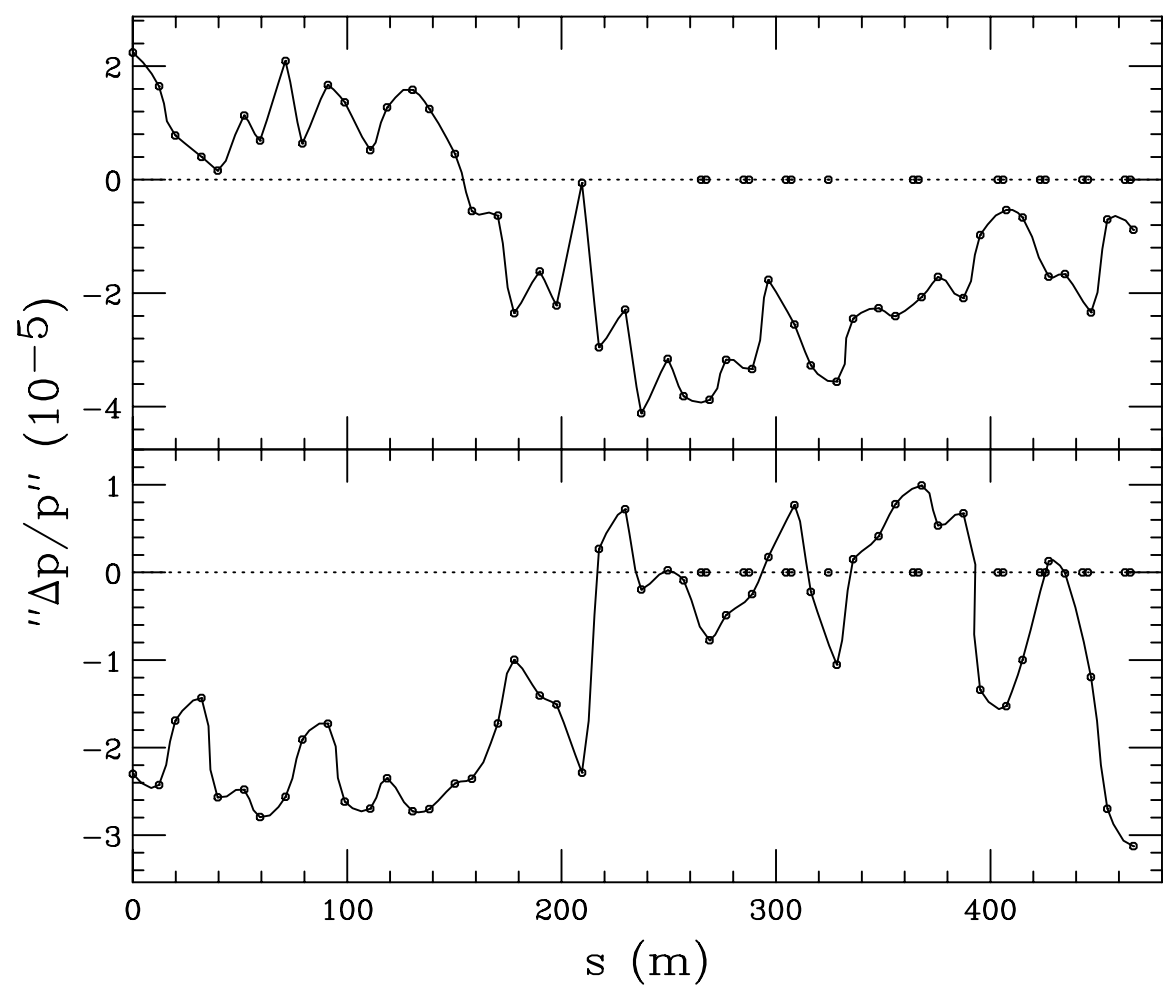

FIG. 14. The spatial function of the synchrotron modes shown in Fig. 13 divided by the dispersion function. The resulting function can be thought of as the amplitude of the off-momentum deviation at each BPM location. The locations of rf cavities are shown as dots on the horizontal axis.

to obtain a new set of fitting parameters, where $\mathbf{I}$ is the identity matrix and $\lambda$ is an adjustable nonnegative parameter to control the behavior of the algorithm.

This fitting scheme works well with MAD simulation data. But the result obtained from the real experimental data does not converge to a reasonable model, probably because the BPM resolution of about $100 \mu \mathrm{m}$ is too large so that the measured data contain too much error for accelerator modeling (see Figs. 4 and 5). The modeling method could be more useful for electron machines where the BPM resolution is about $1 \mu \mathrm{m}$. Combining the ICA derived optical functions with the orbit response matrix modeling, one may be able to provide better constraints on accelerator modeling.

\section{SUMMARY}

In this study we carried out simulations to study the performance and limitation (BPM noise and the number of digitizing turns) of the ICA and PCA methods on data analysis. We find that the ICA can resolve coupled modes that PCA cannot resolve. We have studied the effect of BPM noises and a finite number of sampling points. We find that the ICA method is more robust in mode separation and is less affected by the number of sampling points. The two methods have a similar behavior under low level random noises. We showed that ICA has potential in the study of nonlinear resonances. The meaning of the nonlinear spatial function has not been fully studied.

The main goal of this paper is to use the ICA method to analyze experimental turn-by-turn data of the Fermilab Booster. The method enables us to measure the betatron and synchrotron tunes throughout the fast ramping cycle. We observed an interesting phenomenon that the spatial function of synchrotron modes crosses zero within one turn around the accelerator. The resulting " $\Delta p / p_{0}$ " is about $10^{-5}$. This means that the beam has different fractional off momentum at each BPM position. We believe that this may have resulted from either the longitudinal feedback system or the mismatch between the energy gain in rf cavities and the dipole ramping curve of a fast ramping accelerator.

Finally, the measured beta functions, phase advance, linear coupling angle, and dispersion function can be used for accelerator modeling in conjunction with the orbit response matrix method. The additional constraint on the measured phase advance and linear coupling angle can be incorporated in the orbit response matrix accelerator modeling.

\section{ACKNOWLEDGMENTS}

This work is supported in part by grants from DE-AC0276CH03000, DOE DE-FG02-92ER40747, and NSF PHY0244793. 
[1] J. Safranek, Nucl. Instrum. Methods Phys. Res., Sect. A 388, 27 (1997).

[2] J. Irwin, C. X. Wang, and Y. T. Yan, Phys. Rev. Lett. 82, 1684 (1999); Chun-xi Wang, Ph.D. thesis, Stanford University, 1999 (unpublished).

[3] Chun-xi Wang, Vadim Sajaev, and Chih-Yuan Yao, Phys. Rev. ST Accel. Beams 6, 104001 (2003).

[4] Y. Yan et al., Report No. SLAC-PUB-10369, 2003.

[5] Aapo Hyvarinen, Juha Karhunen, and Erkki Oja, Independent Component Analysis (John Wiley \& Sons, New York, 2001).

[6] S. Choi, A. Cichocki, and Y. Denville, in Proceedings of the Third International Conference on Independent Component Analysis and Blind Signal Separation, San Diego, CA, 2001, edited by T.-W. Lee, T.-P. Jung, S. Makeig, and T.J. Sejnowskipp, pp. 319-322 (http:// ica2001.ucsd.edu/).

[7] A. Belouchrani, K. Abed-Merain, J.F. Cardoso, and E. Moulines, IEEE Trans. Signal Processing 45, 434 (1997).

[8] Booster lattice model maintained by A. Drodzdin. The authors updated corresponding parameters according to operation settings.

[9] X. Yang and J. MacLachlan, Fermilab Tech-note No. TM2239
[10] H. Grote and F. C. Iselin, The MAD Program, Version 8.1, User's Reference Manual, CERN/SL/90-13(AP), 1991.

[11] J.F. Cardoso and A. Souloumiac, SIAM J. Matrix Anal. Appl. 17, 161 (1996).

[12] S. Choi, A. Cichocki, and A Belouchrani, J. VLSI Signal Process. 32, 93 (2002).

[13] A. Belouchrani and A. Cichocki, Electron. Lett. 36, 2050 (2000).

[14] M. Ellison et al., Phys. Rev. E 50, 4051 (1994); S. Y. Lee et al., Phys. Rev. Lett., 67, 3768 (1991); D. D. Caussyn et al., Phys. Rev. A 46, 7942 (1992); M. Syphers et al., Phys. Rev. Lett. 71, 719 (1993); D. Li et al., Phys. Rev. E 48, R1638 (1993); H. Huang et al., Phys. Rev. E 48, 4678 (1993); Y. Wang et al., Phys. Rev. E 49, 1610 (1994); S. Y. Lee, Phys. Rev. E 49, 5706 (1994).

[15] J. Laskar, Physica (Amsterdam) 67D, 257 (1993).

[16] X. Huang, S. Y. Lee, Eric Prebys, and Chuck Ankenbrandt, in Proceedings of PAC2005: The Particle Accelerator Conference, Knoxville, Tennessee, 2005; Fermilab Technote (to be published).

[17] X. Yang and James MacLachlan, Fermilab Tech-note TM2244.

[18] R. Bartolini, A. Bazzani, M. Giovannoizzi, W. Scandale, and E. Todesco, Report No. CERN SL/95-84, 1984. 